\title{
Modern Methods of Etiotropic Therapy and Preventive Measures in Dogs Suffering Dirofilariasis
}

\author{
Nadezhda Bespalova \\ Professor, Department of Veterinary-Sanitary Examination, \\ Epizootiology and Parasitology, Voronezh State Agrarian \\ University named after Emperor Peter the Great \\ Mitchurin Street, 1, Voronezh, Russia \\ Nadezh.bespalova2014@yandex.ru
}

\author{
Sergey Semenov \\ Head of Department of Veterinary-Sanitary Examination, \\ Epizootiology and Parasitology, Voronezh State Agrarian \\ University named after Emperor Peter the Great \\ Mitchurin Street, 1, Voronezh, Russia \\ ss_ramon@mail.ru
}

\author{
Tatyana Zolotykh \\ Veterinarian of veterinary hospital VetLiga \\ Mitchurin Street, 9A, Voronezh, Russia \\ zlata69mail.ru
}

\begin{abstract}
The paper presents the core findings of a study aimed at therapeutic efficacy of domestic antiparasitics such as Inspector Total C and Helmimax on the basis of moxidectin used for etiotropic therapy and prevention of dirofilariasis in dogs. The dogs were infested by $D$. immitis and $D$. repens nematodes under natural conditions. The microfilariae were identified using blood smear technique, the concentration method (Yastrebov 2004) and the filtration through membrane filters Millipore ${ }^{\circledR}$ (Ireland). An antigen of adult mature dirofilaria was detected using Immuno Run Antigen Detection Kit CANINE HEARTWORM produced by Biogal (Israel).

There were selected 57 dogs of different breeds aged 1 to 14 years. They were spontaneously infested by dirofilariasis in natural conditions and three groups were formed. Dogs from Group I $(n=21)$ placebo were used for negative control. Dogs from Group II $(\mathrm{n}=19)$ were given the drug Inspector Total $C$ once at a dose of $2.5 \mathrm{mg} / \mathrm{kg}$ (spot on). Dogs from Group III $(\mathrm{n}=17)$ were given the drug Helmimax once at a dose of $0.25 \mathrm{mg} / \mathrm{kg}$ (per os). Before and after the introduction of preparations, the animals have gone through clinical and hemolarvoscopic examinations on the $3^{\text {rd }}, 14^{\text {th }}, 30^{\text {th }}, 45^{\text {th }}$ and $60^{\text {th }}$ day.

It was established that domestic antiparasitics Inspector Total $C$ and Helmimax on the basis of moxidectin in the above mentioned doses result in $100 \%$ death of microfilariae on the $3^{\text {rd }}$ day after treatment regardless of the intensity of infection and the type of helminth. When used in mono mode for the period of three months, it leads to complete elimination of imaginal forms. Anthelmintics do not cause side effects and are well tolerated by animals.
\end{abstract}

Key words - dog; zoonosis; dirofilariasis; etiotropic therapy; preventive measures

\section{INTRODUCTION}

The urgency of such dangerous zoonosis as dirofilariasis is explained by the fact of its uneasy treatment and prevention processes. The absence of visible clinical signs of the disease at the initial stage, complexity of diagnosis make it difficult to early recognise the infection which leads to the spread of this disease $[5,10,11$, 14, 23, 24, 27, 29]. Effective drugs which are imported to Russia have become inaccessible for the dogs' owners due to their high cost; ; besides, the range of domestic analogues is rather limited. In this regard the study aimed at available and safe methods of treatment of this disease, including preventive measures is very topical.

According to foreign literature, in countries endemic for dirofilaria, there acts a protocol according to which all dogs that are 8 month old (and not older than that) undergo through screening and, in case the infection is not detected, they go through the prophylactics with macrocyclic lactones on a yearly basis during their whole life. For these purposes the following recommendations can be made: ivermectin at a dose of $6 \mathrm{mcg} / \mathrm{kg}$, milbemycin oxime at a dose of $500 \mathrm{mcg} / \mathrm{kg}$, moxidectin at a dose of $3 \mathrm{mcg} / \mathrm{kg}$ (per os, on monthly basis), and selamectin at a dose of $6 \mathrm{mg} / \mathrm{kg}$ (spot-on). The moxidectin formulation providing extended protection is used in the United States of America, Italy, Spain, Australia [21, 22]. The treatment of this disease is aimed at elimination of adult worms with the subsequent destruction of larval stages. In this regard, the purpose of this work was to study the therapeutic efficacy of domestic etiotropic drugs based on moxidectin to solve the problem of etiotropic therapy and prevention of dirofilariasis in dogs.

\section{MATERIALS AND METHODS OF RESEARCH}

The work was performed in the period from 2011 to 2017 in the Department of Parasitology and Epizootiology of Voronezh State Agrarian University named after Emperor Peter the Great, in private and state veterinary hospital of Voronezh and the Voronezh Region, in animal shelters, nurseries and Police Canine Units. The object of the study was dogs infested by nematodes $\mathrm{D}$. immitis and $\mathrm{D}$. repens in 
natural conditions. Venous blood was used for diagnosing active infection. The microfilariae were identified using blood smear technique, the concentration method [16] and the filtration through membrane filters Millipore ${ }^{\circledR}$ (Ireland) [4] using digital camera Levenhuk T510 NG and ToupView software. The structure features of the head and tail ends, the length and width of the larvae body were taken into account. An antigen of adult mature dirofilaria was detected using Immuno Run Antigen Detection Kit CANINE HEARTWORM produced by Biogal (Israel).

To determine the effectiveness of etiotropic therapy for dirofilariasis in dogs, there were conducted 2 series of experiments using domestic moxidectin preparations: Inspector Total $C$ and Gelmimax. For this purpose 57 dogs of different breeds aged of 1 to 14 years were spontaneously infested by dirofilariasis in natural conditions. Out of this number three groups were formed. Dogs from Group I $(n=21)$ placebo were used for negative control. Dogs from Group II $(\mathrm{n}=19)$ were given the drug Inspector Total $C$ once at a dose of $2.5 \mathrm{mg} / \mathrm{kg}$ (spot on). Dogs from Group III $(\mathrm{n}=17)$ were given the drug Helmimax once at a dose of $0.25 \mathrm{mg} / \mathrm{kg}$ (per os). Before and after the introduction of preparations, the animals have gone through clinical and hemolarvoscopic examinations on the 3rd, 14th, 30th, 45th and 60th day of treatment. The obtained data was statistically processed using BioStat 2009, SPSS Statistics 17.0.

\section{RESEARCH RESULTS}

The number of larvae varied slightly during the experiment within Group I placebo, (Fig. 1). Before the Inspector Total C drug was given to dogs from Group II, the intensity of infection (II) ranged from $14.0 \pm 0.0$ to $1007.8 \pm 2.7$ number of larvae per $\mathrm{ml}$ of blood and the average number within the group amounted to $319.2 \pm 0.8$; the intensity of infection (II) was $100 \%$. On the 3rd day after treatment 8 dogs out of 19 did not suffer from microfilariae (in their blood). With the rest of the dogs the number of larvae decreased to $52.6 \pm 0.6$ and the intensity of infection (II) went down to $42.1 \%$. On the 14 th, 30th and 45th day of studies no microfilariae were detected in any blood sample. However, on the 60th day the microfilariae numbered $46.0 \pm 0.1$ larvae per $\mathrm{ml}$ of blood, and the intensity of infection (II) rose from zero to $26.3 \%$ (Fig. 2).

Before the treatment of dogs from Group III with Helmimax, the II ranged from $62.5 \pm 0.4$ to $906.3 \pm 1.5$ microfilariae per $\mathrm{ml}$ of blood and averaged $391.9 \pm 1.1$ within the group. The IS was equal to $100 \%$. The hemolaroscopic examination of dogs on the $3^{\text {rd }}, 14^{\text {th }}, 30^{\text {th }}, 45^{\text {th }}$ and $60^{\text {th }}$ day after treatment did not reveal any microfilariae; the ES treatment was $100 \%$ (Fig. 3).

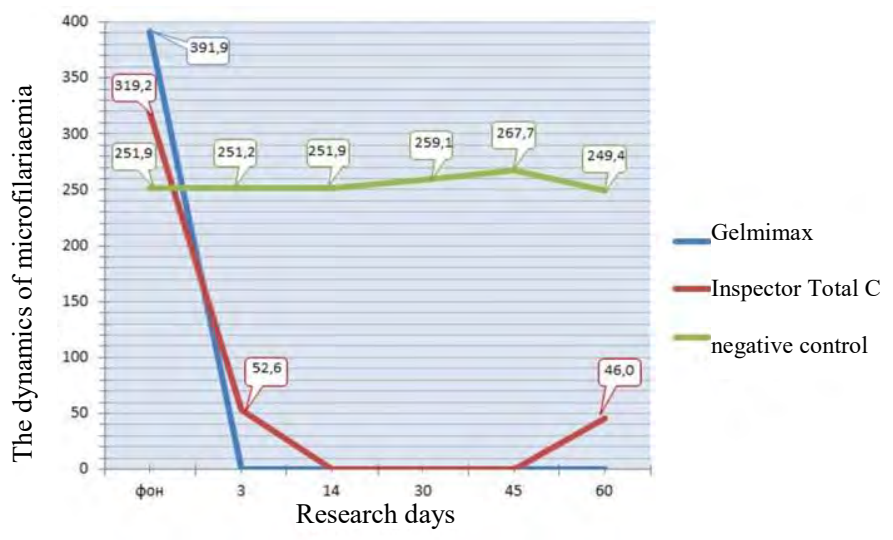

Fig. 1. The dynamics of microfilariaemia in dogs during treatment.

A positive effect was observed in 5 dogs who were identified with microfilariae and to whom the drug was given at a dose of $2.5 \mathrm{mg} / \mathrm{kg}$ (active ingredient: moxidectin) once a month to destroy the imaginal forms of dirofilaria. After 3 months since the last treatment, microfilariae were not detected in the blood; antigenic tests were negative which indicates the elimination of imaginal forms of causative agent. The extensibility of the drug was $62.5 \%$.

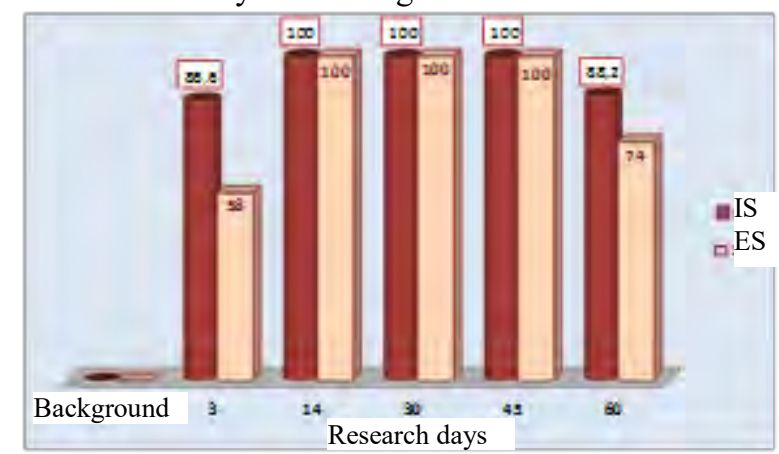

Fig. 2. Dynamics of intensity (IS) and extensibility (ES) during the treatment with Inspector Total C

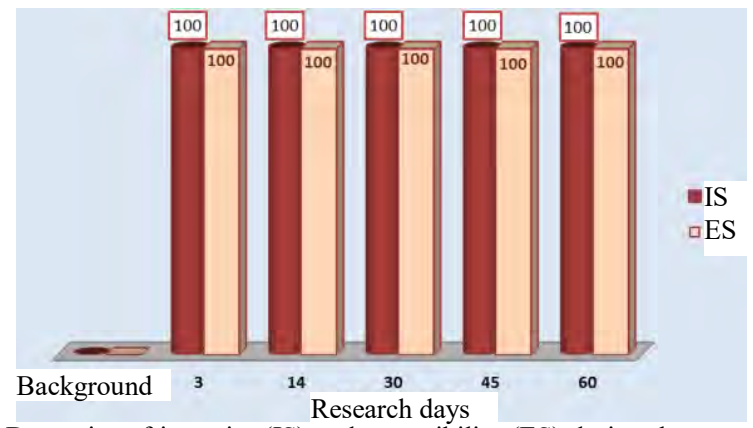

Fig. 3. Dynamics of intensity (IS) and extensibility (ES) during the treatment with Helmimax.

It was established that domestic antiparasitics Inspector Total $C$ and Helmimax on the basis of moxidectin result in $100 \%$ death of microfilariae on the 3rd day after treatment regardless of the intensity of infection and the type of helminth. When used in mono mode, it leads to complete elimination of imaginal forms. Anthelmintics do not cause side effects and are well tolerated by animals. 


\section{DISCUSSION OF RESULTS}

The treatment of disease using macrofilaricides is firstly aimed at the elimination of adult worms, then at the elimination of the larval stages followed by the use of microfilaricides. Melarsomina dihydrochloride (Immititsid), which is a derivative of arsenic, is an effective macrofilaricide nowadays. Melasormin is effective against adult worms and larvae of the $5^{\text {th }}$ stage. According to modern protocol of treatment, it is administered 3 times at a dose of $2.5 \mathrm{mg} / \mathrm{kg}$ of body weight intramuscularly. Its efficiency is $98 \%$ [24, 28]. However, it is necessary to take into account that the use of melarsomin can cause various complications, out of which the most dangerous is pulmonary embolism. During the treatment period dogs should not be able to move freely, they are kept for at least 3 weeks on a leash in a specific place or in cages

Microfilaricidal therapy is carried out 3-6 weeks after the use of macrofilaricides. As a means of microfilaric therapy abroad, macrocyclic lactones are successfully used. In the United States and Italy, where the problem of dirofilariasis is most relevant and well studied, drugs based on ivermectin, moxidectin, milbemycin and selamectin are popular. They have proven to be effective microfilaricides, whose therapeutic efficacy reaches $100 \%$ according to a number of foreign authors. Ivermectin (Endogard, Nuheart, Endex, Biomec, Varmecide, Valuheart, Heartgard) is administered orally at a dose of $6 \mathrm{mg} / \mathrm{kg}$. Selamectin (Revolution, Stronghold) is administered orally at a dose of $6 \mathrm{mg} / \mathrm{kg}$. Moxidectin (ProHeart, Advocate) is administered at a dose of 2.5-6.8 $\mathrm{mg} / \mathrm{kg}$, orally, subcutaneously, or spot-on. Milbemycin (Interceptor, Milbemax) is administered orally at a dose of 500-999 mg/kg [17, 19, 20, 25, 28].

The data on high etiotropic efficacy of Moxidectin and its analogues obtained in the course of this study are consistent with the data of foreign colleagues. The studied domestic drug Helmimax based on moxidectin at a dose of $0.25 \mathrm{mg} / \mathrm{kg}$ of body weight (once, per os) gives 100\% result against both types of microfilariae on the third day of treatment, which lasts 60 days (observation period). While another drug, i.e. Inspector Total C administered in a dose of $2.5 \mathrm{mg} / \mathrm{kg}$ of body weight (once, spot on) gives $100 \%$ result on the $14^{\text {th }}$ day of treatment; however, on the $60^{\text {th }}$ day $1 / 3$ of dogs were diagnosed with microfilariae in their blood. Further treatment of sick dogs with the Inspector Total $C$ drug in a dose of 2.5 $\mathrm{mg} / \mathrm{kg}$ of body weight (once, spot on, once a month) gave positive result. After 3 months since the last treatment, microfilariae were not detected in the blood; antigenic tests showed negative reaction, which indicated the elimination of the imaginal forms of causative agent. In the Russian Federation the list of microfilaricides is limited. The imported drugs, such as, Stronghold, Advocate, Milbemax are expensive and, therefore, inaccessible to many pet owners. Avermectin is used as an active ingredient in domestic medicines. V.F. Postnova et al. [10] and R. S. Arakelyan et al. [1] suggest using the anthelmintic Dironet on the basis of ivermectin, pyrantel pamoate and praziquantel orally at a dose of 1 tablet per $10 \mathrm{~kg}$ of body weight $(6 \mathrm{mg} / \mathrm{kg}$, ivermectin $)$ as an effective domestic microfilaricide. According to the authors, on the $30^{\text {th }}$ day after treatment, complete elimination of parasites was achieved; however, from the $45^{\text {th }}$ to the $60^{\text {th }}$ day microfilariae were identified in the blood; the efficacy of the drug was $98.2 \%$ and $96.3 \%$, respectively. In order to prevent dirofilariasis in dogs, the authors used Dironet monthly at a dose of 1 tablet per $10 \mathrm{~kg}$ of body weight of a dog from April to October.

The studies prove that for the prevention of dirofilariasis in dogs, one can use the domestic drugs based on moxidectin: Helmimax at a dose of $0.25 \mathrm{mg} / \mathrm{kg}$ off body weight (once, per os) and Inspector Total $C$ at a dose of $2.5 \mathrm{mg} / \mathrm{kg}$ of body weight (once, spot on) once a month for a period of three months. Drugs are administered to dogs from the first decade of April to the first decade of October. The dates may be shifted a week in one direction or another, depending on the weather conditions.

Dogs of different breeds, age and gender groups tolerated the drug well. Dakhno Yu. I., Dakhno I. S. [7] used Brotermectin-granulate for etiotropic therapy. As an active ingredient the drug contains $3.2 \mathrm{mg}$ of ivermectin. It is advisable to prescribe it in the early stages of the disease to affect the microfilariae of an early age; ivermectin acts less efficiently at later stages of the larvae or imago and is contraindicated for such dog breeds as collie, sheltie, bobtail and their hybrids. Ivermectin is administered orally at a dose of $1 \mathrm{~g}$ per $10 \mathrm{~kg}$ of body weight, 2 times a day for 5 days in a row. The treatment should be repeated after 56 days. The persistence of the drug is 178 days. It is well tolerated by dogs of all breeds. Studying the microfilaricidal efficacy of three drugs among which were Ivermek, Avertin and Stronghold, M.A. Fisko and E.T. Kepuladze [15] found out that Ivermek had $100 \%$ efficacy in low doses of 0.003 to $0.020 \mathrm{ml} / \mathrm{kg}$ of body weight; however it gave complications in $47.6 \%$ of cases. Stronghold also showed $100 \%$ efficacy against microfilariae in all treated dogs and in no case complications were revealed. The action plan aimed at the fight against dirofilariasis in dogs includes regular hematological studies with timely detection of dogs infected with dirofilariasis and their treatment with Advocate (drug) on monthly basis in doses according to the instruction. Immunoparasitan is indicated in combination with Advocate subcutaneously every third day according to the schedule and in doses following the instruction from the first day of treatment. The authors recommend treating conditionally healthy dogs with Ivermek for prophylactic, curative and recreational purposes at a dose of $0.2 \mathrm{ml}$ per animal (once), then at a dose of $1 \mathrm{ml}$ per $33 \mathrm{~kg}$ of body weight subcutaneously once a month. The treatment should be carried out during the summer period (heavy exposure to mosquitoes): from April through November. S. A. Vedeneev, V.N. Yamschikov [6] proposed therapy against dirofilariasis in three stages: the first is aimed at preparation of the animal for chemotherapy, the second is the chemotherapy itself and the third is rehab and supportive care (rehabilitation). The treatment and rehabilitation scheme developed by the authors is designed for 2 months. The first stage includes immunostimulation with Immunoparasitin five times intramuscularly with an interval of 4 days at a dose depending on body weight. The complex of polysaccharides and lipoproteins activates the T-cell and macrophage systems, which leads to the activation of specific immunocompetent 
cell clones responsible for antiparasitic defense of the body. The second stage of treatment is chemotherapeutic measures aimed at the destruction of parasites. For this purpose, drugs based on ivermectin (Ivomec, Dectomax) are used. On the $24^{\text {th }}$ day from the beginning of the treatment (4 days after the last injection of Immunoparasitan) the Ivomec drug is administered subcutaneously at a dose of $1 \mathrm{ml}$ per $50 \mathrm{~kg}$ of dog weight, three times with an interval of 7 days. The first injection is $80 \%$ of the dose indicated in the manual; the $2^{\text {nd }}$ and the $3^{\text {rd }}$ injections should be injected in full dose. The second stage of the treatment lasts 21 days. The whole period of treatment takes 45 days.

The rehabilitation period lasts 2-3 months during which a balanced diet therapy and moderate exercise are recommended.

The use of the proposed treatment for dogs infected with dirofilariasis makes it possible to reduce the toxic effect of etiotropic drugs and decomposition products of dead parasites. The efficacy of therapeutic measures is $86.3 \%$ and $87 \%$ of dogs remain immune to reinfection with dirofilariasis for 10 months.

Scientists around the world believe that the treatment of dogs infected with dirofilariasis should be a comprehensive and complex approach. Preventive measures in the focus of infection should be aimed at reducing the population of stray definitive hosts and transmission vectors of infection germ, which will prevent the infection of people and domestic animals by dirofilaria. According to foreign literature, in countries endemic for dirofilaria, there acts a protocol according to which all dogs that are 8 month old (and not older than that) undergo through screening and, in case the infection is not detected, they go through the prophylactics with macrocyclic lactones on a yearly basis during their whole life. For these purposes ivermectin in a dose of $6 \mathrm{mcg} / \mathrm{kg}$, milbemycin oxime - $500 \mathrm{mcg} / \mathrm{kg}$, moxidectin - $3 \mathrm{mcg} / \mathrm{kg}$ (per os, monthly), as well as selamectin $-6 \mathrm{mg} / \mathrm{kg}$ (spot-on) are recommended. In Italy, Spain and Australia the injection form of moxidectin is used, which has a prolonged protective effect $[24,26]$.

According to the methodological guidelines for prevention of dirofilariasis operating in the Russian Federation, in places where persistent foci of zoonosis are formed, systematic monitoring of phenology, ecology and species composition of transmission vectors of invasion pathogens is carried out, and the time of their mass departure is determined. An important preventive aspect is aimed at exterminating the larval and adult mosquitoes. Examination and deworming of domestic dogs infected with dirofilariasis should be carried out by veterinary organizations. To prevent dirofilariasis in dogs in the endemic zone, chemoprophylaxis with the same antihelminthics used for treatment is administered. The most convenient are the drugs used no more than 2 times in the mosquito season during the summer months. For example, levamisole, vermitan, dectomax, ivermectin, otodectin, novomek and others are allowed for use for these purposes in the prescribed manner. To prevent the contact of mosquitoes with pets and humans, various long-lasting repellents in the form of emulsion, lotion, spray or powder are recommended [13].

Despite the diversity of existing and emerging chemopreventive agents, the problem of dirofilariasis in our country remains very urgent. In this regard, it is necessary to inform the owners of dogs about the disease, as well as about the need and administration of etiotropic antiparasitic agents.

\section{CONCLUSION}

The studies have shown the possibility of using domestic antiparasitics based on moxidectin: Inspector Total $C$ at a dose of $2.5 \mathrm{mg} / \mathrm{kg}$ of body weight (once, spot on) and Helmimax at a dose of $0.25 \mathrm{mg} / \mathrm{kg}$ of body weight (once, per os) for etiotropic therapy and prevention of dirofilariasis in dogs.

\section{References}

[1] R.S. Arakelyan, The use of Dironet drug with the preventive purpose against service dogs infested with Dirofilaria immitis in the Astrakhan region, Medical parasitology and parasitic diseases, 2011, No. 4, pp. 4142 .

[2] N.S. Bespalova, T.A. Zolotykh, Perspectives of domestic moxidectins application in dirofilariasis carnivorous, Topical issues and methodological approach to diagnosis, treatment and prevention of Animal Diseases: Materials of international scientific and practical conf. - pos. Persianovsky, Donskoy SAU, 2016, pp. 96-100.

[3] N.S. Bespalova, T. Zolotykh, A Domestic moxictectins as etiotropic treatment of dogs' dirofilariasis: Theory and practice against parasitic diseases: Mater. of reports of scientific conf., Moscow, 2016, Issue 17, pp. $68-70$.

[4] A. Bronstein, Filtration methods in the diagnosis of tropical human helminth infections (trematodoses, intestinal nematodoses, filariasis), Medical parasitology and parasitic diseases, 1986, No. 4, pp. 62-66.

[5] A.M. Bronstein, Russia's first autochthonous detection of long-term Dirofilaria repens and the first experience of combined therapy for Dirofilariasis Repens, Epidemiology and infectious diseases, 2013, No. 3, pp. 47-52.

[6] S.A. Vedeneev, V.N. Yamshchikov, New approaches to problems treatment and prevention of dogs' dirofilariasis, Topical issues of biology and veterinary medicine small domestic animals: Proceedings of the International Scientific Practical Conference, Troitsk. SUSAU, 2005, pp. 182-186.

[7] Yu.I. Dakhno, I.S. Dakhno, Microfilaricidal action of Brovermectin granules for the prevention and chemical prophylaxis of dirofilariasis in dogs, Theory and Practice aimed at control of parasitic diseases: Reports of Scientific Conference -Moscow, 2013. - Issue 14. - pp. 130-131.

[8] S. Engashev, The effectiveness of Dironet drug in dirofilariasis of dogs, Veterinarian, 2009, No. 4, p. 13.

[9] N.V. Yesaulova, M.Sh. Akbaev, O.E. Davydova, Diagnostics and therapeutics and preventive measures for dirofilariasis of dogs, Veterinary medicine, 2008, No.2, pp. 30-34.

[10] V.F. Postnova, The most important trend of prevention of dirofilariasis in humans, Theory and practice of parasitic disease control: Reports of Scientific Conference - Moscow, 2011, no. 12, pp. 399 - 401

[11] V.M. Kravchenko, G.S. Itin, D.P. Vinokurova, Infection of domestic and wild carnivorous with Dirofilaria immitis and Dirofilaria repens in various ecological and geographical zones of the Krasnodar Region, Proceedings of Kuban State Agrarian University, 2009, Series: Veterinary Sciences, No. 1 (Part I), pp. 164-165.

[12] M. Fisko, Microfilaricidal therapy for dirofilariasis, Theory and practice of parasitic disease control: Reports of Scientific Conference - Moscow, 2008, Issue 9, pp. 499-501.

[13] B.F. Shulyak, I.A. Arkhipov, Nematodose of dogs (zoonoses and anthropozoonosis, Moscow, 2010, p. 495. 
[14] V.P. Sergiev, The results of study of human dirofilariasis in Russia, Medical parasitology and parasitic diseases, 2014, No. 3, pp. 3-9.

[15] M. Fisko, E. Kepuladze, Dirofilariasis in dogs -prevention and control, Proceedings of Kuban State Agrarian University, 2009, No. 1 (part 1), 2009, pp. $187-188$.

[16] V.B. Yastreb, A comparative study of detection methods of microfilariae in the blood of dogs, Theory and practice of parasitic disease control: Reports of Scientific Conference - Moscow, 2004, Issue 5, pp. 443-445.

[17] C. Bazzocchi, M. Mortarino, G. Grandi, Combined ivermectin and doxycycline treatment has microfilaricidal and adulticidal activity against

[18] Dirofilaria immitis in experimentally infected dogs, Int. J. Parasitol., 38: 1401-1410, 2008.

[19] B. Blagburn, J. Vaughan, Evaluation of susceptibility of heartworm (Dirofilaria immitis) biotypes to microfilariae-based single- dose and dose- mortality regression assays, Processing of the AAVP 56th Annual Meeting, St.Louis, MO, 2011, p. 110.

[20] C. Bourguinat, K. Keller, H. Bhan, Macrocyclic lactone resistance in Dirofilaria immitis, Processing of the AAVP 56th Annual Meeting, St.Louis, MO, 2011, pp. 108.

[21] D.D. Bowman, Heartworms, macrocyclic lactones, and the specter of resistance to prevention in the United States, Parasites Vectors, vol. 5, pp. 138, 2012.

[22] M.T. Dzimanski, J.W. McCall, A.M. Mansour, The effect of prednisone on the efficacy of melarsomine dihydrochloride against adult Dirofilaria immitis in experimentally infected beagles, In State of the Heartworm'10 Symposium, Memphis, T.N. American Heartworm Sosaety, 2010.

[23] M. Malos, A.M. Alho, S.P. Owen, Parasite control practices in companion animals: a survey of dogs and cat owners, Prev. Vet. Med., vol. 122, pp. 174-180, 2014.

[24] J. W. McCall, The safety-net story about macrocyclic lactone heartworm preventatives: A review, an update and recommendations: Veterinary Parasitology, Vol. 133, pp. 197-206, 2005.

[25] J.W. McCall, C. Gendi, I. H. Kramer, Heartworm disease in animals and human, Adv. Parasitol., vol.66, pp. 193-285, 2008.

[26] M. W. Miller, Heartworm disease - the clinical essentials Proceeding of the NAVC North American Veterinary Conference, Jan. 8-12, 2005, Orlando, Florida, pp. 848-849.

[27] B. Menn, S. Lorentz, T.S. Naucke, Imported and travelling dogs as cariers of canine vector-borne pathogens in Germany, Parasit.Vectors, vol. 3, pp.34, 2010.

[28] C.T. Nelson, J. W. McCall, D. Carithers, Current canine guidelines for the prevention, diagnosis, and management of Heartworm (Dirofilaria immitis) infection in dogs, American Heartworm Society, 2014.

[29] D. Traversa, A. Di Cesare, G. Conboy, Canine and feline cardiopulmonary parasitic nematodes in Europe: emerging and underestimated», Parasitol. Vectors, Vol.3, pp. 62, 2010. 